\title{
Two Results on Real Zeros of Chromatic Polynomials
}

\author{
F.M. Dong* \\ Mathematics and Mathematics Education \\ National Institute of Education \\ Nanyang Technological University, Singapore 637616 \\ K.M. Koh \\ Department of Mathematics \\ National University of Singapore, Singapore 117543
}

August 14, 2003

\begin{abstract}
This note presents two results on real zeros of chromatic polynomials. The first result states that if $G$ is a graph containing a $q$-tree as a spanning subgraph, then the chromatic polynomial $P(G, \lambda)$ of $G$ has no non-integer zeros in the interval $(0, q)$. Sokal conjectured that for any graph $G$ and any real $\lambda>\Delta(G), P(G, \lambda)>0$. Our second result confirms that it is true if $\Delta(G) \geq\lfloor n / 3\rfloor-1$, where $n$ is the order of $G$.
\end{abstract}

Keywords: chromatic polynomial, zeros, $q$-tree, simplicial vertex

${ }^{*}$ Corresponding author. Email: fmdong@nie.edu.sg. 


\section{Introduction}

For any graph $G$, let $V(G), E(G), v(G), e(G), \Delta(G)$ and $P(G, \lambda)$ denote, respectively, its vertex set, edge set, order, size, maximum degree and chromatic polynomial.

Given a positive integer $q$, the class of $q$-trees is defined recursively as follows. Any complete graph $K_{q}$ is a $q$-tree, and any $q$-tree of order $n+1$ is a graph obtained from a $q$-tree $G$ of order $n$, where $n \geq q$, by adding a new vertex and joining it to each vertex of a $K_{q}$ in $G$. Thus a graph is a 1 -tree if and only if it is a tree. Let $\mathcal{S}_{q}$ denote the family of graphs containing $q$-trees as spanning subgraphs. Thus $\mathcal{S}_{1}$ is the family of connected graphs. It is known (see [3], for instance) that if $G \in \mathcal{S}_{1}, n=v(G) \geq 2$, then $(-1)^{n-1} P(G, \lambda)>0$ for all real $\lambda$ in the interval $(0,1)$. We extend this observation to the following result.

Theorem 1 Let $G \in \mathcal{S}_{q}$ with $n=v(G) \geq q \geq 1$. Then

$$
P(G, \lambda) \neq 0,
$$

for all non-integer real $\lambda$ in $(0, q)$.

Sokal [4] showed that for any graph $G$, all (real or complex) zeros of $P(G, \lambda)$ lie in the disc $|z|<7.963907 \Delta(G)$. Thus there exists a constant $c$ with $1 \leq c \leq 7.963907$ such that $P(G, \lambda)>0$ for all real $\lambda>c \Delta(G)$. He also conjectured that if $G$ is any graph and $\lambda>\Delta(G)$, then $P(G, \lambda)>0$.

As the second result of this note, we show that Sokal's conjecture is true if $\Delta(G) \geq \frac{1}{3} v(G)-1$.

Theorem 2 If $G$ is a graph of order $n$ and $\lambda>\max \{\Delta(G),\lfloor n / 3\rfloor-1\}$, then $P(G, \lambda)>0$. 


\section{The first result}

Let $G$ be a graph. Given $x, y \in V(G)$ with $x y \in E(G)$, let $G-x y$ denote the graph obtained from $G$ by deleting the edge $x y$, and $G \cdot x y$ be the graph obtained from $G$ by identifying $x$ and $y$, and replacing multi-edges (if they arise) by single ones. The Fundamental Reduction Theorem (see [3], for instance) states that

$$
P(G, \lambda)=P(G-x y, \lambda)-P(G \cdot x y, \lambda)
$$

for every edge $x y$ in $E(G)$.

For any vertex $x$ in a graph $G$, let $N(x)$ denote the set of its neighbours, and $d(x)$ its degree. A vertex $x$ in $G$ is said to be simplicial if either $d(x)=0$ or $N(x)$ forms a clique in $G$. It is known that every $q$-tree, except $K_{q}$ and $K_{q+1}$, contains at least two non-adjacent simplicial vertices. The following result is useful.

Lemma 1 If $u$ and $v$ are two non-adjacent vertices of a q-tree $G$, then $G \cdot u v \in \mathcal{S}_{q}$.

Proof. The result is trivial if $v(G)=q, q+1, q+2$. Let $G$ be a $q$-tree of order $n$, where $n \geq q+3$, and let $u, v$ be two non-adjacent vertices of $G$. If $u$ or $v$, say $u$, is a simplicial vertex of $G$, then $G-u$ is a $q$-tree and also a spanning subgraph of $G \cdot u v$. Thus $G \cdot u v \in \mathcal{S}_{q}$. Assume that both $u$ and $v$ are not simplicial vertices of $G$. Let $w$ be a simplicial vertex of $G$. Observe that $G-w$ is a $q$-tree of order $n-1$. By the induction hypothesis, $(G \cdot u v)-w=(G-w) \cdot u v \in \mathcal{S}_{q}$. This then implies that $G \cdot u v \in \mathcal{S}_{q}$, as required. 
Proof of Theorem 1. Since a $q$-tree always contains a $(q-1)$-tree as a spanning subgraph, to prove Theorem 1, it suffices to show that

$$
(-1)^{n-q} P(G, \lambda)>0
$$

for all real $\lambda$ in $(q-1, q)$.

If $n=q$, then $G=K_{q}$, so that $P(G, \lambda)=\lambda(\lambda-1) \cdots(\lambda-q+1)$, and the result holds trivially.

Assume that $n>q$. Suppose that the result fails. Then there exists a graph $G \in \mathcal{S}_{q}$ of minimum size such that

$$
(-1)^{n-q} P(G, \lambda) \leq 0
$$

for some real $\lambda$ with $q-1<\lambda<q$.

Observe that $G$ cannot be a $q$-tree; otherwise,

$$
P(G, \lambda)=\lambda(\lambda-1) \cdots(\lambda-q+1)(\lambda-q)^{n-q},
$$

and we have

$$
(-1)^{n-q} P(G, \lambda)>0
$$

for $q-1<\lambda<q$.

Let $T$ be a spanning $q$-tree of $G$. Since $G \neq T$, there exists $u v \in E(G)$ such that $u v \notin E(T)$. Thus, by (1),

$$
P(G, \lambda)=P(G-u v, \lambda)-P(G \cdot u v, \lambda),
$$

and we have

$$
(-1)^{n-q} P(G, \lambda)=(-1)^{n-q} P(G-u v, \lambda)+(-1)^{n-1-q} P(G \cdot u v, \lambda) .
$$


By our choice of $G$, we have $G-u v \in \mathcal{S}_{q}$. Also, by Lemma 1, $T \cdot u v \in \mathcal{S}_{q}$, implying that $G \cdot u v \in \mathcal{S}_{q}$. By the minimality of $e(G)$, the result (2) holds for both $G-u v$ and $G \cdot u v$. But then by (3), we have

$$
(-1)^{n-q} P(G, \lambda)>0
$$

for $q-1<\lambda<q$, a contradiction. The result thus follows.

\section{The second main result}

For any subset $S$ of $V(G)$, let $G \cdot S$ denote the graph obtained from $G$ by identifying all vertices in $S$ and replacing all multi-edges by single ones. We first state the following known result which will be used in the proof that follows.

Lemma $2([\mathbf{1}, \mathbf{2}]) \quad$ Let $x y$ be an edge of a graph $G$. Then

$$
\begin{aligned}
& P(G, \lambda)-(\lambda-d(x)) P(G-x, \lambda) \\
= & P(G-x y, \lambda)-(\lambda-d(x)+1) P(G-x, \lambda) \\
& +\sum_{\substack{u \in N(x) \backslash\{y\} \\
y u \notin E(G)}} P(G \cdot\{x, y, u\}, \lambda) .
\end{aligned}
$$

To prove Theorem 2, we prove the following stronger result.

Theorem 3 Let $G$ be a graph and suppose $V(G)$ is partitioned into $A$ and $B$, where $|A|=a \geq 0$ and $|B|=b \geq 0$. Let $\Delta_{A}=\max \left\{d_{G}(x): x \in A\right\}$. Then for $\lambda>\max \left\{\Delta_{A},\lfloor a / 3\rfloor+b-1\right\}$,

(i) $P(G, \lambda)>0$; and 
(ii) for any $x \in A$,

$$
P(G, \lambda) \geq\left(\lambda-d_{G}(x)\right) P(G-x, \lambda),
$$

where the equality holds if and only if $x$ is a simplicial vertex.

Proof. If $a=0$, the assertions (i) and (ii) hold vacuously. Assume that both (i) and (ii) hold when $a<k$, where $k \geq 1$. Now let $a=k$. By the induction hypothesis, assertion (i) holds for the graph $G-x$, where $x \in A$, i.e., $P(G-x, \lambda)>0$ for

$$
\lambda>\max \left\{\Delta_{A},\lfloor a / 3\rfloor+b-1\right\} \geq \max \left\{\Delta_{A \backslash\{x\}},\lfloor(a-1) / 3\rfloor+b-1\right\} .
$$

As $\lambda-d(x)>0$, assertion (ii) implies assertion (i). Hence it suffices to prove that assertion (ii) holds.

Let $x$ be any vertex in $A$. If $x$ is a simplicial vertex, then (ii) holds, since

$$
P(G, \lambda)=(\lambda-d(x)) P(G-x, \lambda) .
$$

Thus (ii) also holds when $d(x) \leq 1$.

Assume that (ii) holds when $d(x)<s$, where $s \geq 2$. Now assume that $x$ is not simplicial and $d(x)=s$. Let $w \in N(x)$. Since the degree of $x$ in $G-x w$ is $s-1$, assertion (ii) holds for $G-x w$, i.e.,

$$
P(G-x w, \lambda)-(\lambda-d(x)+1) P(G-x, \lambda) \geq 0
$$

for $\lambda>\max \left\{\Delta_{A},\lfloor a / 3\rfloor+b-1\right\}$.

Then, by Lemma 2 and (4), assertion (ii) holds if $N(x) \backslash(N(w) \cup\{w\}) \neq \emptyset$ and

$$
P(G \cdot\{x, w, u\}, \lambda)>0
$$


for $\lambda>\max \left\{\Delta_{A},\lfloor a / 3\rfloor+b-1\right\}$ and for every $u \in N(x) \backslash(N(w) \cup\{w\})$. We shall prove them below.

Since $x$ is not a simplicial vertex, there exist two non-adjacent vertices in $N(x)$. So if $w$ is selected to be one of such vertices, then $N(x) \backslash(N(w) \cup$ $\{w\}) \neq \emptyset$.

Let $u$ be any vertex in $N(x) \backslash(N(w) \cup\{w\})$ and let $H=G \cdot\{x, w, u\}$. Suppose $v$ is the resulting vertex in $H$ after contracting $x, w$ and $u$ in $G$. Let $B^{\prime}=(B \backslash\{w, u\}) \cup\{v\}$ and $A^{\prime}=A \backslash\{x, w, u\}$. Observe that $\left|A^{\prime}\right|<|A|=a$ and $A^{\prime} \cup B^{\prime}$ is a partition of $V(H)$. By the induction hypothesis, $P(H, \lambda)>0$ for $\lambda>\max \left\{\Delta_{A^{\prime}},\left\lfloor\left|A^{\prime}\right| / 3\right\rfloor+\left|B^{\prime}\right|-1\right\}$. So (5) holds if

$$
\max \left\{\Delta_{A^{\prime}},\left\lfloor\left|A^{\prime}\right| / 3\right\rfloor+\left|B^{\prime}\right|-1\right\} \leq \max \left\{\Delta_{A},\lfloor a / 3\rfloor+b-1\right\} .
$$

Notice that $\Delta_{A^{\prime}} \leq \Delta_{A},\left|A^{\prime}\right| \leq a-1$ and $\left|B^{\prime}\right| \leq b+1$. But if $\left|B^{\prime}\right|=b+1$, then $w, u \in A$, implying that $\left|A^{\prime}\right|=a-3$. So we always have

$$
\left\lfloor\left|A^{\prime}\right| / 3\right\rfloor+\left|B^{\prime}\right|-1 \leq\lfloor a / 3\rfloor+b-1 .
$$

Thus (6) holds.

Therefore assertion (ii) holds. This completes the proof.

By letting $B=\emptyset$, Theorem 2 now follows from Theorem 3. We shall end this paper by giving the following remarks.

\section{Remarks:}

(i) Theorem 2 verifies Sokal's conjecture only for a special case, namely, the order of $G$ is at most $3 \Delta(G)+5$.

(ii) The method used in the proof of Theorem 3 is unlikely of any use in proving Sokal's conjecture due to the fact that new graphs (the $H$ 's) 
created from the recurrence relation in Lemma 2 may have their maximum degrees greater than that of the original graph. There might be some hope to establish Sokal's conjecture if one could find a recurrence relation for chromatic polynomials which does not produce any new graph with higher maximum degree even after a finite number of iterations.

(iii) In Sokal's conjecture, the maximum degree is at least 3. It may be more realistic to start off the study by considering the extreme case when $\Delta(G)=3$.

(iv) The "maxmaxflow" of a graph $G$, denoted by $\Lambda(G)$, is defined as

$$
\Lambda(G)=\max _{x \neq y} \lambda(x, y)
$$

where

$$
\begin{aligned}
\lambda(x, y) & =\text { maximum number of edge-disjoint paths from } x \text { to } y \\
& =\text { minimum number of edges separating } x \text { from } y
\end{aligned}
$$

Since $\lambda(x, y) \leq \min \{d(x), d(y)\}$, we have $\Lambda(G) \leq \Delta(G)$. In his private communication, Sokal also conjectured that for any graph $G$ and $\lambda>$ $\Lambda(G), P(G, \lambda)>0$.

Acknowledgment. The authors are grateful to the referee for his valuable suggestions. 


\section{References}

[1] F.M. Dong, Proof of a Chromatic polynomial Conjecture, Journal of Combinatorial Theory, Ser. B 78, 35-44(2000).

[2] J. Oxley, Colouring, packing and critical problem, Quart. J. Math. Oxford Ser., 29 (1978), 11-22.

[3] R.C. Read and W.T.Tutte, Chromatic polynomials, in: Selected Topics in Graph Theory III (eds. L.W.Beineke and R.J.Wilson), Academic Press, New York (1988), 15-42.

[4] A. D. Sokal, Bounds on the complex zeros of (Di)chromatic polynomials and potts-model partition functions, Combin. Probab. Comput. 10 (2001), 41-77. 